\title{
Modeling and Simulation of Vibrations of Non- Homogeneous Annular Plate of Quadratic Thickness Resting on Elastic Foundation
}

\author{
Robin, N. Dhiman, A. Chauhan, M. Dhiman1
}

\begin{abstract}
Mathematical modeling is presented to analyze natural frequencies of vibrations of an isotropic annular plate of quadratic varying thickness resting on Winkler type elastic foundation where numerical simulation is carried out using quintic spline technique for three different combinations of edge conditions. Effect of elastic foundation, together with nonhomogeneity variation, on the natural frequencies of vibration is illustrated for variety of thickness variation for the first three modes. To compare parametric effect on a specific plate, transverse displacements are presented in normalized form. Accuracy of the results and validity of numerical method is demonstrated by comparing the existing results in the literature.
\end{abstract}

Keywords: Quintic Spline, Elastic Foundation, NonHomogeneity, Annular plate, Quadratic Thickness

\section{INTRODUCTION}

Study of elastic foundation, non-homogeneity and/or solidity, and thickness variation on dynamic response of annular plates has immense importance due to its parts, thus making it necessary to research in this area which will help to improve the design of diaphragms of steam turbines, cylinder heads and piston heads in particular.In practical, modeling of the effect of foundation on structure is quite complex as phenomenon of actual response, and interaction between the system and foundation both are complicated to understand.However, Winkler, Pasternak and Vlasov, etc. are few proposed different types of foundation which approximate the actual behaviour of foundation. In this endeavor Bhattacharya [1] considered triangular plates with Vlasov foundation and Wang and Stephens [2] carried out their findings using Timoshenko beam resting on Pasternak foundation.

Many instances in real world such as highways with reinforced concrete pavements, building's foundation slabs and airport runways, etc., prove Winkler type foundation as more prominent foundation which is actually based on the virtual replacement of real foundation by a progression of vertical springs exclusive of shear interaction under the theorythat it manifests the proportional deflection at each point.Many researchers (in 1970's and 1980's)started considering Winkler type foundation in their work, few are mentioned. Chonan [3] considered rectangular plates to analyze the natural frequencies while plates are assumed on 'Winkler elastic foundation'.

Revised Manuscript Received on August 10, 2019.

Robin, N. Dhiman, Department of Mathematics, Graphic Era Hill University, Dehradun, India.

A. Chauhan, Graphic Era Deemed to be University, Dehradun, India.

M. Dhiman, Department of Mathematics, Graphic Era Hill University, Dehradun, India. application in theconception and structure of machinery

Gupta et al., [4] studied the consequence of "Winkler" type foundation on vibrations of annular pate where the thickness is varying linearly, and Tomar et al.[5] carried their research oncircular plates and studied the behavior of frequencies and frequency modes of free vibration with the effect of a Winkler type foundation.

Due to technical advancement where fibre-reinforced materials are highly used to maintain the flexibility under any possible operation, for instance use of diaphragms formed by different materials in pressure capsules, switches etc, it becomes necessary to examine the consequences of non-homogeneity on the vibrational behavior of annular plates. Non-homogeneity is also considered by the researchers in various ways in different models [6-10].The presented work here deals with exponential variation of nonhomogenity under the consideration of parameter $\mu$ in radial directionwhich is different from the density parameter $\eta$ in the same direction. This provides a justified model as compared to the models where nonhomogeneity for Young's modulus variation $E=E_{0} e^{\mu x}$, and density variation $\rho=\rho_{0} e^{\eta x}$ are assumed in similar conduct.

Thus under all above consideration, a "mathematical model is formulated to study the vibrational behaviour of a non homogeneous annular plate of quadratic varying thickness which is resting on a Winkler type elastic foundation using quintic spline interpolation technique which provides the numerical simulation under three edge conditions (at inner and outer radii) namely $(\mathrm{C}-\mathrm{C}),(\mathrm{C}-\mathrm{F})$, and $(\mathrm{C}-\mathrm{S})$, here the symbols $\mathrm{C}, \mathrm{F}$ and $\mathrm{S}$ stand for clamped, free and simply supported respectively".

\section{METHODOLOGY}

Considering an isotropic non-homogeneous annular plate (inner and outer radii $\mathrm{b}$ and a) of thickness $h(r)$, referred to a system of cylindrical coordinates $(\mathrm{r}, \theta, \mathrm{z})$. Then the mathematical model -fourth order partial differential equation- represents the phenomenon is as follows:

$$
\begin{aligned}
& D \frac{\partial^{4} w}{\partial r^{4}}+\frac{2}{r}\left[D+r \frac{\partial D}{\partial r}\right] \frac{\partial^{3} w}{\partial r^{3}}+\frac{1}{r^{2}}\left[-D+r(2+v) \frac{\partial D}{\partial r}+r^{2} \frac{\partial^{2} D}{\partial r^{2}}\right] \frac{\partial^{2} w}{\partial r^{2}}+\frac{1}{r^{3}}\left[D-r \frac{\partial D}{\partial r}+r^{2} v \frac{\partial^{2} D}{\partial r^{2}}\right] \frac{\partial w}{\partial r} \\
& +\rho h \frac{\partial^{2} w}{\partial t^{2}}+K_{f} w=0,
\end{aligned}
$$

where $D=\frac{E h^{3}}{12\left(1-\nu^{2}\right)}, \mathrm{D}, w, E(r)$ and $K_{f}$ are flexural rigidity, transverse deflection,Young's modulus and foundation constant, respectively. 
To introduce non-homogeneity in plate material, let us assume that $E$ and $\rho$ are functions of space variable r. Now equation (1) becomes

$$
\begin{aligned}
& E h^{3} \frac{\partial^{4} w}{\partial r^{4}}+\frac{2}{r}\left[E h^{3}+r\left(h^{3} \frac{d E}{d r}+3 E h^{2} \frac{d h}{d r}\right)\right] \frac{\partial^{3} w}{\partial r^{3}} \\
& \left.+\frac{1}{r^{2}}\left[\begin{array}{l}
-E h^{3}+r(2+v)\left(h^{3} \frac{d E}{d r}+3 E h^{2} \frac{d h}{d r}\right) \\
+r^{2}\left(h^{3} \frac{d^{2} E}{d r^{2}}+6 h^{2} \frac{d E}{d r} \frac{d h}{d r}+3 E\left(2 h\left(\frac{d h}{d r}\right)^{2}+h^{2} \frac{d^{2} h}{d r^{2}}\right)\right)
\end{array}\right)\right] \begin{array}{l}
\frac{\partial^{2} w}{\partial r^{2}} \\
\frac{r^{2}}{2}
\end{array} \\
& \left.+\frac{1}{r^{3}} E h^{3}-r\left(h^{3} \frac{d E}{d r}+3 E h^{2} \frac{d h}{d r}\right)+r^{2} v\left(\begin{array}{c}
h^{3} \frac{d^{2} E}{d r^{2}}+6 h^{2} \frac{d E}{d r} \frac{d h}{d r} \\
+3 E\left(2 h\left(\frac{d h}{d r}\right)^{2}+h^{2} \frac{d^{2} h}{d r^{2}}\right)
\end{array}\right)\right]\left(\frac{\partial w}{\partial r}\right. \\
& +12 \rho h\left(1-v^{2}\right) \frac{\partial^{2} w}{\partial t^{2}}+K_{f} w=0 .
\end{aligned}
$$

Using $\quad x=\frac{r}{a}, \bar{w}=\frac{w}{a}, \bar{h}=\frac{h}{a}$ equation (2) is non-dimensionalise and thickness quadratic variation is expressed as, $\bar{h}=h_{0}\left(1+\alpha x+\beta x^{2}\right)$, inner edge, taper parameters are represented by $\alpha$ and $\beta$. Material non-homogeneity is assumed as

$E=E_{0} e^{\mu x}, \rho=\rho_{0} e^{\eta x}$,

where, ${ }^{\mu}$ and $\eta_{\text {are non-homogeneity parameters and }} \rho_{0}$ is the density and $E_{0}$ is Young's Modulus at the inner edge.

$$
\bar{w}(x, t)=W(x) e^{i \xi t},
$$

where $\xi$, radian frequency, provides harmonic motion and lead Eq. (2) to a liner ordinary differential equation

$$
F_{0} \frac{d^{4} W}{d x^{4}}+F_{1} \frac{d^{3} W}{d x^{3}}+F_{2} \frac{d^{2} W}{d x^{2}}+F_{3} \frac{d W}{d x}+F_{4} W=0
$$

where,

$$
\begin{aligned}
& F_{0}=1 \\
& F_{1}=2(1+Q x) / x \\
& F_{2}=Q^{2}+R+\{(2+v) Q / x\}-\left\{1 / x^{2}\right\} \\
& F_{3}=\left\{(1-Q x) / x^{3}\right\}+\left\{v\left(Q^{2}+R\right) / x\right\} \\
& F_{4}=-\left\{\Omega^{2} e^{(\eta-\mu) x} / P^{2}\right\}+\left\{\left(12\left(1-v^{2}\right) E_{f} / e^{\mu x} A^{3} h_{0}^{3}\right)\right\} \\
& \Omega^{2}=12 \rho_{0} a^{2} \xi^{2}\left(1-v^{2}\right) / E_{0} h_{0}^{2} \\
& E_{f}=K_{f} a / E_{0}, \\
& P=1+\alpha x+\beta x^{2}, Q=\mu+\{(3 \alpha+6 \beta x) / P\} \\
& R=\left(6 \beta-3 \alpha^{2}-6 \beta^{2} x^{2}-6 \alpha \beta x\right) / P^{2} .
\end{aligned}
$$

Eq.(4), which is quite complex to solve as it involves several plate parameters and thus it necessities to find a numerical such that $|\alpha| \leq 1,|\beta| \leq 1$ and $\alpha+\beta>-1$, where $h_{0}$ is the thickness at

solution.Therefore further study is carried out using spline interpolation technique along with computer programming in order to get the results of desired accuracy. To make it possible boundary condition are imposed at the inner edge of annular plate, $X=\varepsilon$, and at the outer edge $X=1$.

In this method,

$$
\begin{aligned}
& \bar{W}(X)=\sum_{i=0}^{4} a_{i}\left(X-X_{0}\right)^{i}+\sum_{j=0}^{n-1} b_{j}\left(X-X_{j}\right)_{*}^{5} \\
& \text { where }\left(X-X_{j}\right)_{*}=\left\{\begin{array}{cc}
0, & X \leq X_{j} \\
X-X_{j}, & X>X_{j}
\end{array}\right.
\end{aligned}
$$

A shape function and its first four derivatives resembles $\mathrm{W}(\mathrm{x})$ and respective derivatives because $\bar{W}(X)$ so approximately represent the deflection function for free vibration, in the domain $[\varepsilon, 1]$, where $\Delta X=(1-\varepsilon) / n$, and $X_{k}=k \Delta X,(k=0(1) n)$.

Thus eq.(5) helps us to reduce equation (4) in the form of

$$
\begin{aligned}
& F_{4} a_{0}+\left[F_{4}\left(X_{k}-X_{0}\right)+F_{3}\right] a_{1}+\left[F_{4}\left(X_{k}-X_{0}\right)^{2}\right. \\
& \left.+2 F_{3}\left(X_{k}-X_{0}\right)+2 F_{2}\right] a_{2}+\left[F_{4}\left(X_{k}-X_{0}\right)^{3}+3 F_{3}\left(X_{k}-X_{0}\right)^{2}\right. \\
& \left.+6 F_{2}\left(X_{k}-X_{0}\right)+6 F_{1}\right] a_{3}+\left[F_{4}\left(X_{k}-X_{0}\right)^{4}+4 F_{3}\left(X_{k}-X_{0}\right)^{3}\right. \\
& \left.+12 F_{2}\left(X_{k}-X_{0}\right)^{2}+24 F_{1}\left(X_{k}-X_{0}\right)+24 F_{o}\right] a_{4} \\
& +\sum_{j=0}^{n-1}\left\{\begin{array}{l}
F_{4}\left(X_{k}-X_{0}\right)_{*}^{5}+F_{3}\left(X_{k}-X_{0}\right)_{*}^{4}+20 F_{2}\left(X_{k}-X_{0}\right)_{*}^{3} \\
+60 F_{1}\left(X_{k}-X_{0}\right)_{*}^{2}+120 F_{o}\left(X_{k}-X_{0}\right)_{*}
\end{array}\right\} b_{j}=0 .
\end{aligned}
$$

Eq. (6) generates undetermined homogeneous system which can be express in a matrix representation

$[\mathbf{A}][\mathbf{B}]=[\mathbf{0}]$

where, "A" is coefficient matrixof dimension $(n+1) \times(n+5)$ along with matrix " $\mathrm{B}$ " which is a column matrix contains $\mathrm{n}+5$ unknowns $\left(\mathrm{a}_{\mathrm{i}}\right.$ 's and $\left.\mathrm{b}_{\mathrm{j}}, \mathrm{s}\right)$.

For complete specification of mathematical model discussed above, appropriate boundary conditions must be imposed Therefore, three cases of boundary conditions namely clamped-clamped, clamped-simply supported and clampedfree edge are considered.

Applying boundary conditionson both the edgesusing the expression $W=\frac{d W}{d X}=0 ; W=\frac{d^{2} W}{d X^{2}}+(v / X) \frac{d W}{d X}=0$; and $\frac{d^{2} W}{d X^{2}}+(v / x) \frac{d W}{d X}=\frac{d^{3} W}{d X^{3}}+(1 / X) \frac{d^{2} W}{d X^{2}}-\left(1 / X^{2}\right) \frac{d W}{d X}=0$

for clamped, simply supported and free edge, respectively, four additional equations will emerge with $(n+5)$ unknownsfor different set of boundary and can be expressed via matrix representation as

$$
[\mathrm{C}][\mathrm{B}]=[0]
$$

Thus the Eq. (7) and Eq.(8) collectively produce a consistent system $\quad\left[\frac{\mathbf{A}}{\mathbf{C}}\right][\mathbf{B}]=[\mathbf{0}]$.

Eq. (9), offers a non-trivial solution when the characteristic determinant of above system vanishes, i.e. 
$\left|\frac{\mathbf{A}}{\mathbf{C}}\right|=\mathbf{0}$

\section{RESULTS AND DISCUSSION}

Equation (10) providesthree different sets of frequency equations, for differentchoice of boundary conditions, to get the“values of frequency parameter $\Omega$ "under a choice of plate parameters. Numerical simulation of vibrations of annular plates with three particular thickness variation $\alpha=-0.5 ; \beta=-0.1 ; \alpha=0.0 ; \beta=0.0$; and

$\alpha=+0.5 ; \beta=+0.1$; is carried out for the discussion and these plates are name as Type-I, Type -II and Type-III. Natural frequencies of first threemodes for these three platesare computed and presented for the permissible range of other plate parameters such as elastic foundation $E_{f}=0.0(0.1) 0.5$; non-homogeneity

parameter

$\mu=-0.3(0.1) 0.3 ; \quad$ density

parameter

$\eta=0.0(0.1) 0.5$; for all three edge conditions. During numerical computation, stability and accuracy of results up to four decimal places achieved by running computer programme for dividing the domain into different subintervals. In all the computation $n=400$ has been fixed which leads more stable and reliable results (Tables 1) as compared with existing results in literature.
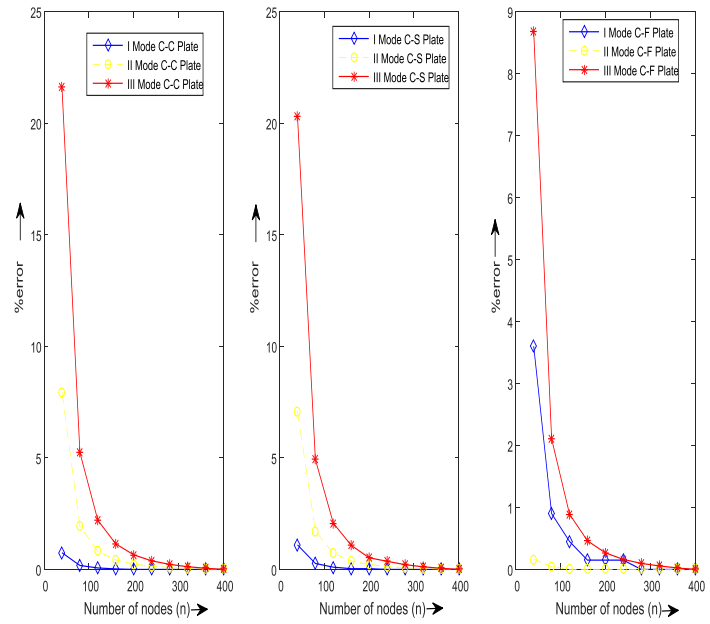

Figure 1.Stability analysis: Error versesnumber of subintervals (n) , (a) I-mode (b) II- mode (c) III- mode, for $\alpha=0.0, \beta=0.0 ; \diamond C$-C plate , $\odot \mathrm{C}-\mathrm{S} ; * \mathrm{C}-\mathrm{F}$ edge; Percentage error $=\left[\left(\Omega_{n}-\Omega_{400}\right) / \Omega_{400}\right] \times 100 ; n=40(40) 400$.
Table 1Comparative resultsfor uniform $(\alpha=0.0, \beta=0.0)$ homogeneous $(\eta=0.0, \mu=0.0)$, isotropic annular plate with $\varepsilon=0.3$

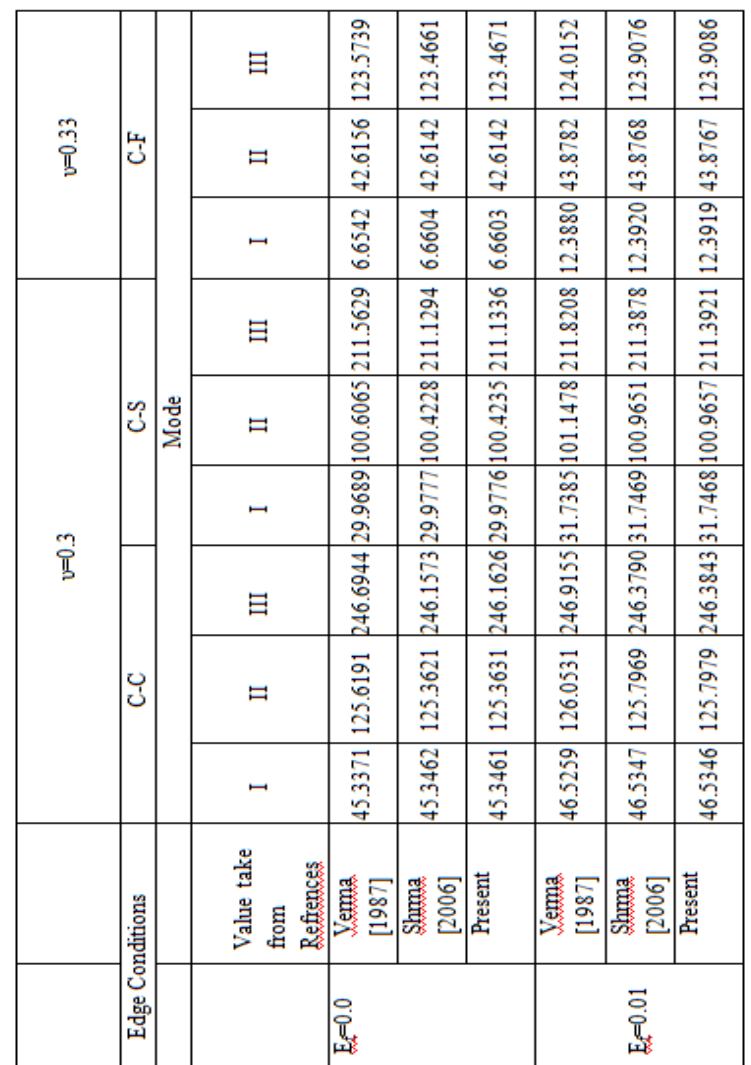

Overall it has been observed that values of $\Omega$ for Type-II (Uniform) plate is smaller than Type-I( $\alpha=-0.5 ; \beta=-0.1 ;)$ and greater than for Type-III( $\alpha=+0.5 ; \beta=+0.1 ;$ ) plate irrespective of other plate parameters. While value of $\Omega$ keeps onincreasingwith the risingvalues of elastic foundation $E_{f}$, non-homogeniety parameter $\mu$ and taper parameters $\alpha$ and $\beta$, it decreases with density parameter $\eta$.

Figures (2-4) show the parametric change for frequency parameter along withelastic foundation, non-homgeneity parameter and density parameter respectively.

From figure $2(\mathrm{a}, \mathrm{b}, \mathrm{c})$, Increasing behavior of $\Omega$ is been observed with increasing value of $E_{f}$ when plate vibrates in first, second and third mode, respectively. This rise in the value of $\Omega$ is subject to thickness variation and keeps on increasing with increasing value of $\alpha$ or $\beta$ or both; irrespective of edge conditions of the plates. Also it is more obvious in case of plate vibrating in first mode as compared to second and third mode. The rate of increase of $\Omega$ is highest for C-F plate than that of other modes and edge conditions.

Figure 3(a) represents the change in frequency with respect to the change in density parameter $\eta$ without compromising non-homogeneity of the material keeping $\mu=0.5$. 
Decreasing trend has been observed in frequency with the escalating value of density or solidity parameter $\eta$. A similar tendency is noticed for second as well as third modes figure 3(b) and 3(c), however the rate of change in frequency parameter varies with thickness and mode of vibration as well. A surprising fact has been observedin case of C-F plate, frequencies with respect to thickness variation is just reverse in second and third mode that of first mode of vibration.

Figure $4(a, b, c)$ depict the effect of non-homogeneity variation, with the parametric value of $\mu$, and its influence on first three frequencies and modesfor the fixed value of density parameter keeping as $\eta=0.5$. An Increment blueprint has been presented for $\Omega$ and $\mu$ with the variation of thickness parameters $\alpha$ and $\beta$.

Nature of frequency parameter $\Omega$ astonishes under the edge condition C-F. Plate takes opposite (unexpected) shape in first mode with thickness variation.

Figure 5, 6 and 7 show the graphical represents of nodal circles for all three types of plates namely Type-I ( $\alpha=-0.5, \beta$ $=-0.1)$, Type-II $(\alpha=0.0, \beta=0.0)$ and Type-III $(\alpha=0.5, \beta=0.1)$ of composite material $(\mu=0.5, \eta=0.5)$ for ClampedClamped, Clamped -Simply supported and Clamped-Free plates, respectively. As the outer edge become thicker, nodal circles shift towards inner edge irrespective of boundary conditions. Clamped-Free edge plate behaves differently in first modes as illustrated by its normalized displacement,

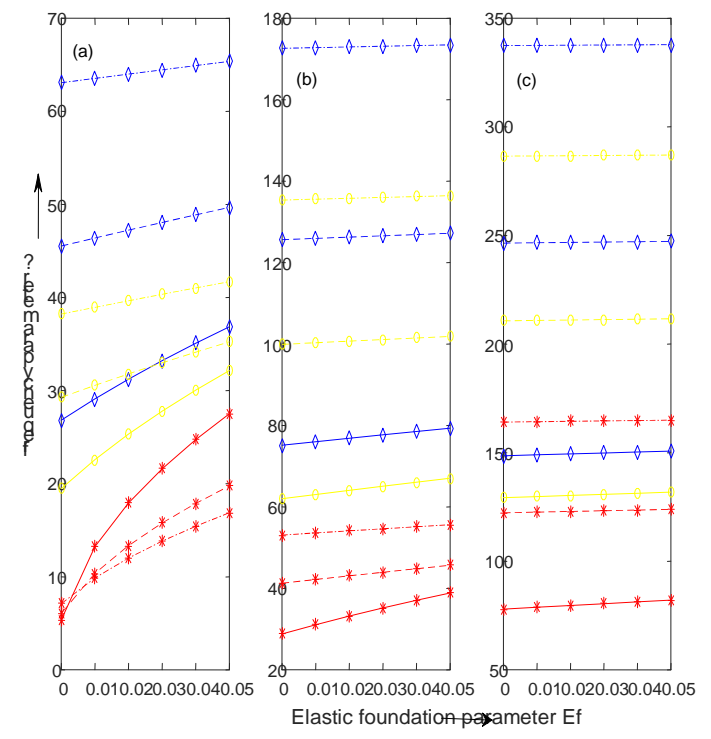

Figure 2. Variation in natural frequencies (a) I-mode (b) II- mode (c) III- mode,with elastic foundation when $\mu=0.5$ and $\boldsymbol{\eta}=0.5$, with edge conditions: " $\nabla \mathrm{C}$-C plate ; $\circ \mathrm{C}-\mathrm{S}$ plate; *C-F edge;" for different thickness: ",$- \alpha$ $=-0.5$. $\beta=-0.1 ;----, \alpha=0.0$. $\beta=0.0 ;,-.-.-\alpha=0.5$. $\beta=0.1$ "
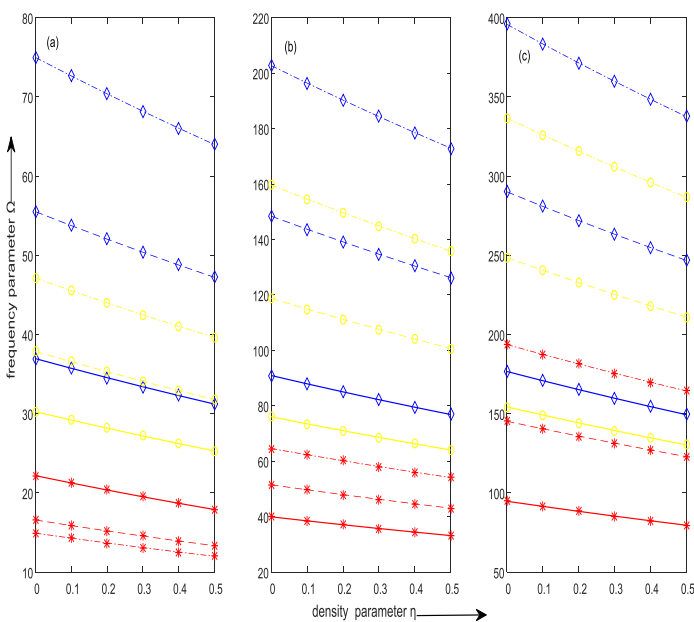

Figure 3 Variation in natural frequencies (a) I-mode (b)

II- mode (c) III- mode with density parameter for $E_{\mathrm{f}}=0.02$ and $\boldsymbol{\mu}=0.5$, with edge conditions: " $\vee \mathrm{C}$-C plate , $\circ \mathrm{C}-\mathrm{S}$ plate; *C-F edge"; for different thickness: ",$- \alpha$ $=-0.5$. $\beta=-0.1 ;---, \alpha=0.0 . \beta=0.0 ;-.-. \alpha=0.5, \beta=0.1$ ".
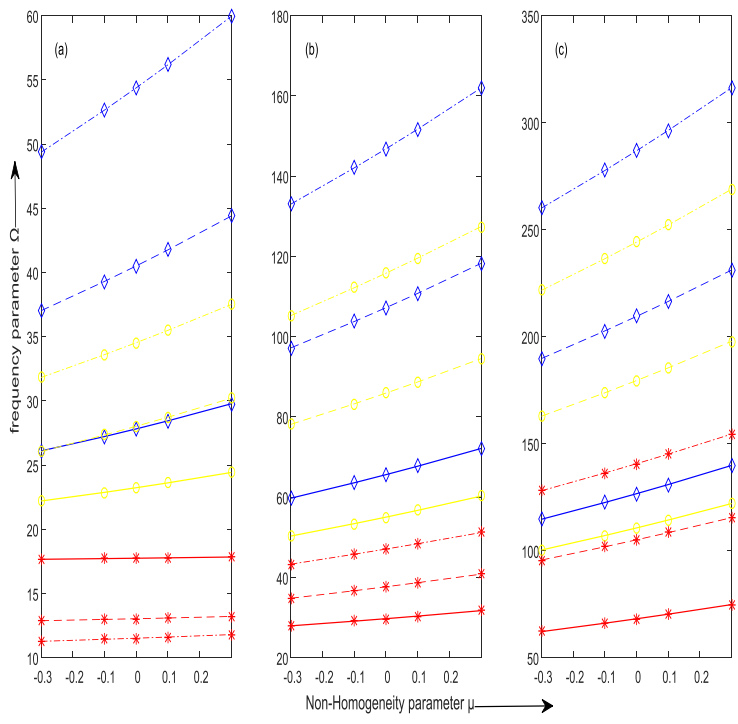

Figure 4. Variation in natural frequencies (a) I-mode

(b) II- mode (c) III- mode, with non-homogeniety parameter when $\mathrm{Ef}=0.02$, and $\eta=0.5$, with edge conditions: " $\diamond \mathrm{C}$-C plate ,, $\mathrm{C}$-S plate $* \mathrm{C}$-F edge"; for different thickness: ",$- \alpha=-0.5$. $\beta=-0.1 ;---, \alpha=0.0$. $\beta=0.0 ;,-. .-\alpha=0.5$. $\beta=0.1$;" 


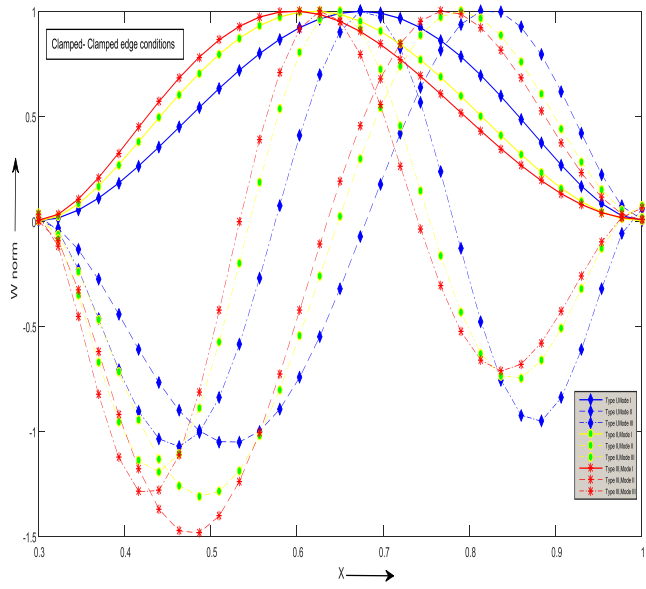

Figure 5. Nodal lines for $\mathrm{C}-\mathrm{C}$ edge conditions, for $\boldsymbol{\mu}=\mathbf{0 . 5}$, $\eta=0.5$; for "—, I- mode;-—,II- mode, .-.-.,IIImode;"with thickness variation: " $\diamond$, (TYPE-I, $\alpha=-0.5, \beta=-$ 0.1); o, (TYPE-II, $\alpha=0.0, \beta=0.0)$; *, (TYPEIII, $\alpha=0.5, \beta=0.1)$ ".

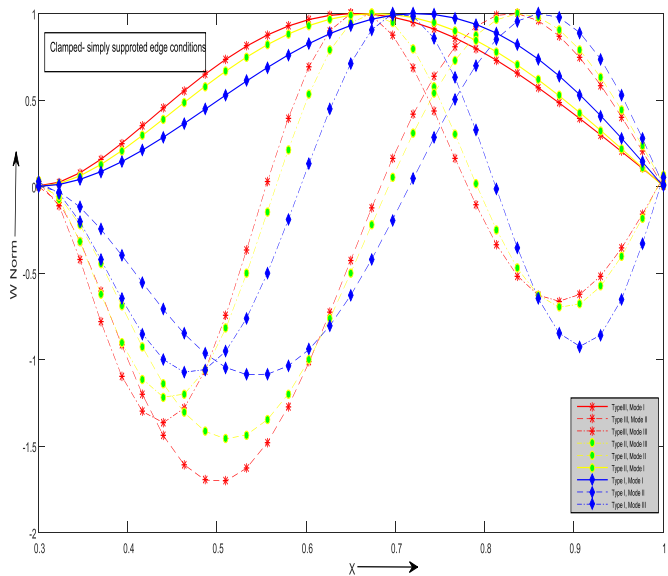

Figure 6.Nodal lines for $\mathrm{C}-\mathrm{S}$ edge conditions, for $\boldsymbol{\mu}=\mathbf{0 . 5}$, $\eta=0.5$ for "-, I- mode; -, ,II- mode, .-.-.,III-

mode;" with thickness variation: " $\diamond,($ TYPE-I, $\alpha=-0.5, \beta=$. 0.1); o, (TYPE-II, $\alpha=0.0, \beta=0.0)$; *, (TYPEIII, $\alpha=0.5, \beta=0.1) "$.

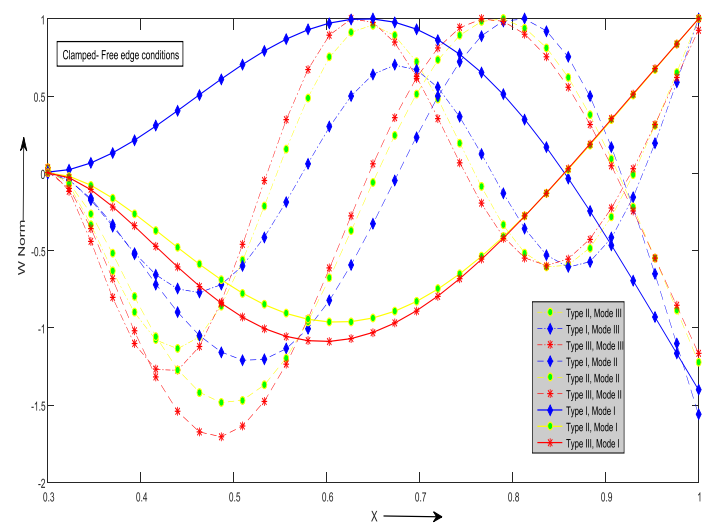

Figure 7.Nodal lines for $\mathrm{C}-\mathrm{F}$ edge conditions, for $\boldsymbol{\mu}=\mathbf{0 . 5}$, $\eta=0.5$ for "-, I- mode; - -,II- mode, .-.-.,III-

mode;"with thickness variation: " $\diamond$,(TYPE-I, $\alpha=-0.5, \beta=$ 0.1); o, (TYPE-II, $\alpha=0.0, \beta=0.0) ; *$, (TYPEIII, $\alpha=0.5, \beta=0.1)$ ".

\section{CONCLUSION AND FUTURE SCOPE}

In the present work more complicating effects on natural frequencies and transverse modes of annular plates are accumulated and presented which will help design engineers to construct more reliable products and will motivate to rethink of their findings and constructions in this era of advancement. Hence, present modeling can be used as benchmark for annular plates and many other results can be evaluated for different choice of thickness variations (linear, parabolic, quadratic), for homogeneous/non-homogeneous with or without foundation.

\section{REFERENCES}

1. B. Bhattacharya, "Free vibration of plates on Vlasov's foundation", J. Sound Vib., Vol.54, 1977, pp. 464-467.

2. \ T. M. Wang, J. E. Stephens, "Natural frequencies of Timoshenko beams on Pastemak foundation", J. Sound Vib., Vol .51, 1977, pp. 149-155.

3. S. Chonan, "Random vibration of an initially stressed thick plate on an elastic foundation”,J.Sound Vib, Vol. 71, 1980, pp.117-127.

4. U. S. Gupta,R. Lal and C.P. Verma, "Effect of an elastic foundation on axisymmetric vibrations of polar orthotropic annular plates of variable thickness", J. Sound Vib., Vol. 103, 1985, pp. 159-169.

5. J. S. Tomar, D. C. Gupta and V. Kumar,"Freevibrations of nonhomogeneous circular plates of variable thickness resting on an elastic foundation",J.Engg. Design, Vol.1, 1983, pp. 49-54.

6. U.S. Gupta, R. Lal, S. Sharma, "Vibration analysis ofnonhomogeneous circular plate of nonlinear thickness variation by differential quadrature method", J. Sound Vib., Vol. 298, 2006, pp.892-906.

7. R. Lal, Dhanpati, "Transverse vibrations of non homogeneous orthotropic rectangular plates of variable thickness: a spline technique", J. Sound Vib., Vol.306, 2007, pp. 203-214.

8. S.Sharma,U.S. Gupta, P.Singhal, "Vibration Analysis of NonHomogeneous Orthotropic Rectangular Plates of Variable Thickness Resting on Winkler Foundation”, J. Appl. Sci. Engg,, Vol.15, 2012, pp. 291-300.

9. U.S. Rana, Robin, "Damped vibrations of parabolic tapered nonhomogeneous infinite rectangular plate resting on elastic foundation", Int. J Engg., Vol. 28, 2015, pp.1082-1089.

10. U.S. Rana, Robin, "Effect of Damping and Thermal Gradient on Vibrations of Orthotropic Rectangular Plate of Variable Thickness", Appl. Appl. Math., Vol.12, 2017, pp.201-216 